Results Four models to engage children as patient-partners have been identified: (1) Moving from family-centred care to child-centred care; (2) Using a triadic approach involving the healthcare professional, parent and child; (3) Using family-centred care while acknowledging the vulnerability of the child; (4) Involving the child patient on a level of participation based on cognitive development and competency. Throughout the analysis process, we identified ethical challenges that can occur when using the different models. Establishing trust with children and addressing issues related to power are the two most prevalent ethical challenges reported.

Conclusions The presentation will focus on these two key ethical challenges and highlight the different perspectives (i.e. from children, parents and healthcare providers) present in the qualitative literature on how to build trust with children in health services planning and delivery.

\section{WOMEN'S ACCOUNTS OF ALTERED FETAL MOVEMENT: UNRELIABLE AND PRIVILEGED}

J Clark. University of Leicester, UK

\subsection{6/bmjopen-2021-QHRN.7}

Background Sociological literature has reported widely on the subjugation of pregnant women's embodied knowledge to obstetric technologies. Women's accounts of altered fetal movement have sometimes been dismissed, with poorlymanaged episodes of reduced fetal movement highlighted as an important contributory factor to avoidable stillbirth. NHS England's 2016 Saving Babies' Lives care bundle included recommendations for the management of reduced fetal movement as part of a national strategy to reduce stillbirth.

Aim To explore how different forms of knowledge about fetal movement are evaluated and prioritised before, during and after the clinical encounter.

Methods This research used an ethnographic approach to compare practice relating to fetal movement at two UK maternity units, with over 200 hours of observation, interviews, and document analysis. Field notes, interview transcripts, policy documents, maternity notes and clinical guidelines were analysed to identify key themes.

Results Despite no cases of women's accounts being dismissed, many clinicians presented altered fetal movement as a highly subjective and unreliable symptom. Women's reports of a quiet baby were not always consistent with clinicians' own impressions of fetal movement, or with the evidence generated by 'objective' monitoring devices. Nonetheless, in accordance with clinical guidance, if a woman reported altered fetal movement at or beyond 39 weeks' gestation, the decision to expedite the birth through induction of labour was regularly made based on her account alone.

Conclusions In the risk-averse maternity setting, and amid a national campaign to reduce stillbirth, clinicians are under considerable pressure to act promptly on women's reports of altered fetal movement, whether or not they trust them. This imperative to act without clear evidence of fetal compromise can be interpreted as a disruption to the established knowledge system, with women's embodied knowledge apparently being privileged - in this scenario at least - over the evidence generated by high-status obstetric technologies.

\section{8 'DEALING WITH THE BABY YOU LOST AND THE LOSS OF THE FUTURE': CHI - A RECURRENT PLACENTAL CONDITION}

E Cornish, D Williams, S Tariq. University College London, UK

\subsection{6/bmjopen-2021-QHRN.8}

Background Chronic histiocytic intervillositis (CHI) is a rare, poorly-understood placental condition causing pregnancy loss. Unlike many other causes of stillbirth, CHI has a recurrence rate of $50-80 \%$ in future pregnancies and has limited treatment options.

Objectives To understand the impact of CHI on women who have experienced pregnancy loss and to ascertain their healthcare needs.

Methods We conducted a focus group discussion (FGD) with three women affected by CHI, recruited through a Facebook support group. The FGD was facilitated by the lead author and a peer-researcher. It was transcribed verbatim and analysed thematically. It is part of a larger clinical study (MIRAPO) which was approved by the London Research Ethics Committee (19/LO/0105).

Results Lack of knowledge about CHI amongst healthcare providers (HCP) often resulted in inadequate or inaccurate explanation of the condition; limited advice on future treatment options; and lack of patient information. This led women to lose trust in HCPs, feeling that they had to seek and arrange their own care, often drawing upon a network of other women with $\mathrm{CHI}$.

The recurrent nature of CHI is particularly challenging, causing women to lose trust in their ability to carry a baby. They described a 'double grief': firstly, for their loss of their baby; secondly, for the loss of the future they had envisaged. The high likelihood of recurrence provoked anxieties about future pregnancies, including unproven treatment regimens and the possibility of further loss. Participants identified continuity of care from HCPs (e.g. bereavement midwives, or an obstetrician familiar with $\mathrm{CHI}$ ) as an important source of hope and support.

Conclusions Lack of awareness around CHI leads to inaccurate information, misplaced reassurance, and failure to refer for appropriate management. This erodes trust and has significant psychological sequelae. In response to these findings, we are developing resources on $\mathrm{CHI}$ for patients and providers.

\section{COMMUNICATION OF UNCERTAINTY, DIAGNOSIS AND TRUST}

${ }^{1} \mathrm{M}$ Dahm, ${ }^{2} \mathrm{C}$ Crock. ${ }^{1}$ Institute for Communication in Health Care, College of Arts and Social Sciences Australian National University, Australia; ${ }^{2}$ The Royal Victorian Eye and Ear Hospital, Melbourne, Australia

\subsection{6/bmjopen-2021-QHRN.9}

Background Diagnosis is a complex and challenging clinical and communicative task. The rate of diagnostic errors, including missed, delayed, and misdiagnosis, has remained prevalent at $10-15 \%$ over the last few decades (Graber, 2013). Diagnostic errors can be a major cause of loss of trust in the doctorpatient relationship. Clinicians use 'diagnostic statements' to name, describe or explain the health problem to patients. If uncertain, clinicians are encouraged to share their working diagnosis and give an indication to their level of uncertainty. 